\title{
Bibliographie der Publikationen der Soncino-Gesellschaft
}

Eine erste Bibliographie, die alle bis zu diesem Zeitpunkt erschienenen Publikationen der Soncino-Gesellschaft verzeichnete, war bereits 1931 von Julius Rodenberg $^{1}$ erarbeitet worden.

Abraham Horodisch hatte $1963^{2}$ in seinen Erinnerungen eine Auflistung aller von der Soncino-Gesellschaft herausgegebenen Publikationen erstellt. Horodisch hatte 83 Titel aufgelistet ${ }^{3}$, wobei er auf geschäftliche Mitteilungen, interne Rundschreiben, Einladungen, Tischkarten etc. verzichtete. Horodisch gab auch die oft umfänglichen Druck- und Widmungsvermerke an sowie Beschreibungen zur äußeren Gestaltung. Wiederabgedruckt wurde seine Bibliographie 1967 in der Zeitschrift Imprimatur ${ }^{4}$, gekürzt um die Druckvermerke.

Die vorliegende Bibliographie umfasst 119 Einträge und ist ein überarbeiteter und ergänzter Wiederabdruck der vom Kölner Antiquar Ulrich Heider 2006 veröffentlichten Bibliographie der Schriften der Soncino-Gesellschaft. Heiders Bibliographie erschien in einem kleinen Band ${ }^{5}$ über die Soncino-Gesellschaft, der als Privatdruck in 500 nummerierten Exemplaren anlässlich der Kabinettsausstellung Die Soncino-Gesellschaft der Freunde des jüdischen Buches e.V. (1924-1937) im Rahmen der 2. Kölner Antiquariatstage von ihm veröffentlicht worden war. Seine Bibliographie lehnt sich im Aufbau sehr eng an Abraham Horodischs Bibliographie an, verzeichnet jedoch insgesamt 106 Titel.

Die vorliegende Bibliographie übernimmt Heiders Systematik, wurde jedoch neudurchnummeriert. Bei jeder bibliographischen Beschreibung wurdevermerkt, unter welcher Nummer der jeweilige Titel bei Horodisch und Heider angeben ist. Auf die äußere Beschreibung der einzelnen Titel, die sowohl bei Horodisch als auch Heider zu finden ist, wurde verzichtet. Da die Bände ursprünglich mit Interimseinbänden versehen an die Mitglieder geliefert wurden und nach dem

1 Rodenberg, Julius: Die Soncino-Gesellschaft der Freunde des jüdischen Buches e.V., Berlin. In: Deutsche Bibliophilie in drei Jahrzehnten. Verzeichnis der Veröffentlichungen der deutschen bibliophilen Gesellschaften und der ihr gewidmeten Gaben 1898-1930, herausgegeben von der Deutschen Bücherei. Leipzig 1931, S. 199-210.

2 Horodisch, Abraham: Ein Abenteuer im Geiste. Die Soncino-Gesellschaft der Freunde des jüdischen Buches. In: Joost, Siegfried (Hrsg.): Bibliotheca Docet. Festgabe für Carl Wehmer. Amsterdam 1963, S. 198-208.

3 Bei den Nummern 1, 10, 19, 31, 55 und 73 vergab Horodisch auch a und $b$.

4 Horodisch, Abraham: Die Soncino-Gesellschaft der Freunde des jüdischen Buches. In: Imprimatur, Neue Folge V (1967), S. 143-148.

5 Heider, Ulrich: Die Soncino-Gesellschaft der Freunde des jüdischen Buches e.V. (1924-1937). Köln 2006, S. 41-80. 
jeweiligen Geschmack des einzelnen Sammlers von ihm neu gebunden werden sollten, ist das äußerliche Erscheinungsbild ein und desselben Werkes heutzutage oft sehr unterschiedlich. Die Herausgeberinnen entschieden sich daher für den Abdruck einer Bibliographie im engeren Sinne.

\section{Ordentliche Veröffentlichungen}

1

Sefer Meschalim, genannt das Kuhbuch, das ist eine Sammlung von Fabeln und Parabeln aus den Büchern Maschal-ha-Kadmoni und Mischle Schualim. Ausgewählt und in jüdisch-deutsche Reime gebracht von Moses Wallich aus Worms mit Holzschnitten versehen und gedruckt in Frankfurt a.M. bei Johannes Wust im Jahre 1687. Berlin 1925. 58 Blätter. [Text in jiddischer Sprache]

Druckvermerk: Faksimile-Reproduktion hergestellt als Privatdruck für die Mitglieder der Soncino-Gesellschaft. Chanukah 5685 [1925]. Erste Publikation der Soncino-Gesellschaft der Freunde des jüdischen Buches e.V., Berlin. Ein zweiter Band, enthaltend Übersetzung des Werkes und Beiwort von Prof. Dr. A. Freimann ist in Vorbereitung. Horodisch 1a, Heider 1

\section{2}

Die Fabeln des Kuhbuches in Übertragung. Mit einem Vorwort von Prof. Dr. Aron Freimann. Berlin 1926. XVI, 97 Seiten.

Druckvermerk: Sefer Meschalim [in hebr. Buchstaben], das Buch der Fabeln, auch Kuhbuch genannt, eine Sammlung von Fabeln und Parabeln aus Maschal-ha Kadmonim und Mischle Schualim, von Mose ben Eli'eser Wallich ausgewählt und in jüdisch-deutsche Reime gebracht, wurde von der Soncino-Gesellschaft der Freunde des jüdischen Buches e.V., Berlin, als ihre erste Publikation im Faksimiledruck nach der im Jahre 1697 zu Frankfurt am Main erschienen Ausgabe zu Chanukka 5685 (das ist Dezember 1925) herausgegeben. Als Beiheft zur ersten Publikation erschienen im Februar 1926 die Fabeln des Kuhbuches in Übertragung von Dr. R. Beatus und mit einem Vorwort von Prof. Dr. A. Freimann als Druck der Offizin W. Drugulin, Leipzig in Janson-Type. Horodisch 1b, Heider $1 a$

\section{3}

S[amuel] J[oseph] Agnon. Das Schass meines Großvaters. Berlin 1925. 13 Blätter.

Druckvermerk: Zweite Publikation der Soncino-Gesellschaft der Freunde des jüdischen Buches e.V. Übersetzt von E.M. Das hebräische Original erschien in: Festskrift 
i anledning af Professor David Simonsens 70-aarige fødselsdag, Kopenhagen 1923, S. 17ff. Hergestellt für die Mitglieder in 550 Exemplaren aus Anlaß des Jahrestages der Gründung der Gesellschaft von Marx \& Co., Berlin, Lag beomer 5685 / Mai 1925. Horodisch 2, Heider 2

4

Arnold Zweig. Die Umkehr des Abtrünnigen. Schauspiel in fünf Akten. Berlin 1925. 97 Seiten.

Druckvermerk: Druck der Ernst Ludwig Presse. Als dritte Publikation der SoncinoGesellschaft der Freunde des jüdischen Buches e.V., Berlin, im Sommer 1925 in 500 Exemplaren hergestellt. Horodisch 3, Heider 3

5

Suezkint. Der Jude von Trimberg. Minnelieder. Berlin 1926. 6 Blätter.

Druckvermerk: Der Text nach der einzigen Niederschrift in der Manessischen Liederhandschrift zu Heidelberg folgt der Ausgabe Friedrich Heinrich von der Hagens. Vierte Publikation der Soncino-Gesellschaft der Freunde des jüdischen Buches. Gedruckt im Januar MCMXXVI bei Gebr. Mann, Berlin, in 800 Exemplaren. Horodisch 4, Heider 4

6

Verzeichnis der auserlesenen Büchersammlung des seeligen Herrn Moses Mendelssohn. [Nachdruck der Ausgabe] Berlin 1786. [Nachbemerkung von Herrmann Meyer.) Berlin 1926. 56, V Seiten.

Druckvermerk: Als fünfte Publikation der Soncino-Gesellschaft der Freunde des jüdischen Buches e.V., Berlin wurde zum 5. September 1926 dieser Prof. Dr. Heinrich Loewe-Druck durch eine Spende von Mitgliedern des Vorstandes in 800 Abzügen hergestellt. Den Druck besorgte F.A. Brockhaus, Leipzig. Nachbemerkung von Herrmann Meyer. Horodisch 5, Heider 5

7

Samuel Lewin. Zeitwende. Roman. [Berlin 1926]. 333 Seiten.

Druckvermerk: Von dem bisher unveröffentlichten Roman erscheint der erste Teil in gemeinschaftlich mit dem Verfasser besorgter Übersetzung aus dem Jiddischen als sechste Publikation der Soncino-Gesellschaft der Freunde des jüdischen Buches e.V., Berlin. Gedruckt bei Poeschel \& Trepte, Leipzig, in der Garamond-Antiqua in einer einmaligen Auflage von 800 Exemplaren. Horodisch 6, Heider 6 


\section{8}

Haggadah. Das Fragment der ältesten mit Illustrationen gedruckten Haggadah wurde nach dem Original aus der Sammlung Elkan Nathan Adler, jetzt im Besitze des Theological Seminary zu New York, reproduziert und als Publikation sieben der Soncino-Gesellschaft der Freunde des jüdischen Buches e.V. ihren Freunden und Mitgliedern zu Chanukkah 5687 überreicht. Berlin 1927. 2 Blätter. Horodisch 7, Heider 7

9

Lesebuch für jüdische Kinder. Zum Besten der jüdischen Freyschule. [Nachdruck der Ausgabe:] Berlin in Commission bey Christian Friedrich Voss und Sohn, 1779. Berlin 1927. 46 Seiten.

Druckvermerk: Soncino-Gesellschaft der Freunde des jüdischen Buches. Achte Publikation. Berlin im Juli 1927. Tammus 5687. Druck: Siegfried Alweiss, Berlin. Horodisch 8 , Heider 8

\section{0}

Jakob Steinhardt. Neun Holzschnitte zu ausgewählten Versen aus dem Buche Jeschu ben Elieser ben Sirah mit einer Einleitung von Arnold Zweig. Berlin 1929. 12 Blätter.

Druckvermerk: Diese neunte Publikation der Soncino-Gesellschaft der Freunde des jüdischen Buches e.V. ist dem Andenken an Dr. Siegfried Wolff gewidmet, der als erster den Vorsitz der Gesellschaft geführt hat. Die Auflage beträgt 800 Exemplare auf Bütten. Den Druck besorgte Aldus Druck, Berlin, im Januar 1929. Der hebräische und deutsche Text folgt der von Rudolf Smend veranstalteten Ausgabe der neuaufgefundenen Genisah-Handschrift; für die Genehmigung gebührt dem Verlage von Walter de Gruyter in Berlin Dank. Soweit der hebräische Originaltext nicht erhalten war, wurde die Übersetzung von J.S. Frenkel benutzt. Horodisch 9, Heider 9

11

Gotthold Ephraim Lessing. Die Juden. Ein Lustspiel in einem Aufzug. Berlin 1929. 57 Seiten. [Vgl. 88 und 119]

Druckvermerk: Gotthold Ephraim Lessings Lustspiel: Die Juden wurde als X. Publikation der Soncino-Gesellschaft der Freunde des jüdischen Buches, Berlin, auf der Handpresse der Officina Serpentis in einer Auflage von 600 Exemplaren gedruckt. Vorher erschien das Werk in 200 Exemplaren als Spendendruck des Herm Menko Max Hirsch für die Teilnehmer an der Jahresversammlung 1929 der Soncino-Gesellschaft. Satz und Druck von E.W. und E.H. Tieffenbach. Horodisch 10b, Heider 10 
12

Franz Rosenzweig. Ein Buch des Gedenkens. Berlin 1930. 59 Seiten.

Druckvermerk: Elfte Publikation der Soncino-Gesellschaft der Freunde des jüdischen Buches e.V. Herausgegeben von Herrmann Meyer. Gesetzt in Italienischer Antiqua. In 800 Exemplaren gedruckt von Aldus Druck Berlin. Den Druck stifteten Reinhold und Erich Scholem zur Jahresversammlung in Berlin am 30. März 1930. Horodisch 11, Heider 11

13

(Paul Rieger.) Jüdische Gotteshäuser und Friedhöfe in Württemberg. Herausgegeben vom Oberrat der Israelitischen Religionsgemeinschaft Württemberg. (Der beschreibende Teil der Veröffentlichung ist von dem theologischen Mitglied des Oberrats, Stadtrabbiner Dr. P[aul] Rieger verfasst, die Lichtbildaufnahmen stammen mit wenigen Ausnahmen von Willi Moegle in Stuttgart.) Stuttgart 1932. 142 Seiten.

Druckvermerk: Die Lichtbilder sind mit wenigen Ausnahmen von Willi Moegle, die Klischees von Löffler \& Bock, Atelier für graph. Künste, der Druck durch U. Levi, Graphische Kunstanstalt und Druckerei, sämtlich in Stuttgart, hergestellt, der Anfangsbuchstabe des Vorworts stammt von Alice Garnmann, Berlin. Als zwölfte Publikation der Soncino-Gesellschaft der Freunde des jüdischen Buches e.V. Berlin wurde die vorliegende Sonder-Ausgabe in 700 Exemplaren abgezogen. Horodisch 12, Heider 12

14

Chamischa Chumsche Thorah. Berlin 1930-1933. 2 Bände mit 134 Blättern. Buchschmuck und Typographie von Marcus Behmer.

Druckvermerk (hebräisch, hier in der Übersetzung nach Horodisch): Das Fünfbuch der Lehre, durchgesehen auf Grund der Messorah sowie auf Grund alter Drucke und der Ausgabe von Ginsburg aus dem Jahre 686 [=1926] ist erschienen bei der Soncino-Gesellschaft in Berlin, korrigiert durch Schemuel Meir Jurovics unter Mithilfe von Meir [= Meyer] Gold, Menachem [d.i. Menko Max] Hirsch, Abraham [d.i. Arthur] Spanier. Die Schrift zeichnete Marcus Behmer nach dem Vorbild der Schrift von Gerschom Kohen in Prag. Der Druck dauerte vom Jahre 690 [= 1930] bis 693 [= 1933] in der Druckwerkstatt der Officina Serpentis des E. W. Tieffenbach in Berlin. 850 Exemplaren [sic] wurden auf Van Gelder-Papier gedruckt, 6 auf Pergament auf den Namen der Subskribenten Jehudah Heskel, Zewi [d.i. Herrmann] Meyer, Schelomoh Salman Schocken. Horodisch 13, Heider 13 
15

Rudolf Hallo. Jüdische Kunst aus Hessen und Nassau. Berlin: Soncino-Gesellschaft e.V., 1933. 39, 33 Seiten.

Druckvermerk: Als Sonderdruck aus: „Religiöse Kunst aus Hessen und Nassau“. Kritischer Gesamtkatalog der Ausstellung Marburg 1928. Herausgegeben von Hermann Deckert, Robert Freyhan, Kurt Steinbart im Verlag des Kunstgeschichtlichen Seminars Marburg-Lahn wurden 600 Exemplare dieses Abschnittes ausgegeben als vierzehnte Publikation der Soncino-Gesellschaft der Freunde des jüdischen Buches e.V., Berlin. Horodisch 14, Heider 14

16

Frucht vom Baum des Lebens. Ozer Peroth Ez Chajim. Die Sammlung der Rechtsgutachten Peri Ez Chajim des Rabbinerseminars Ets Haim zu Amsterdam. Zeitlich geordnet, ins Deutsche übertragen und in gekürzter Form herausgegeben von Menko Max Hirsch. Berlin-Antwerpen 1936 [1937]. 368 Seiten.

Druckvermerk: Dieses Buch wurde gedruckt in 1000 Exemplaren für die Mitglieder der „Soncino-Gesellschaft der Freunde des jüdischen Buches“ und den Herausgeber und Übersetzer Herrn Menko Max Hirsch. Der Druck wurde begonnen im Adar (5)692 [hebr.] Februar 1932 begonnen und vollendet im Sebat (5)697 [hebr.] Januar 1937. Horodisch 15, Heider 15

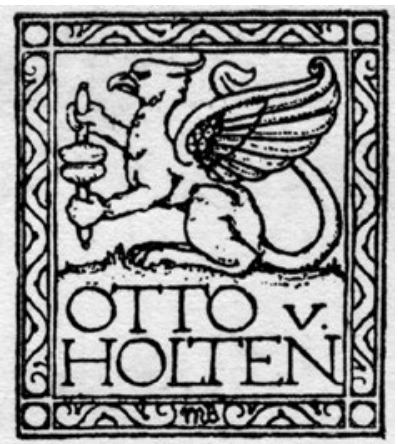

Abb. 40: Druckerzeichen Otto von Holten. 


\section{Sonderpublikationen}

17

Das Buch Judith. Darmstadt: Ernst Ludwig Presse, 1923. 45 Seiten.

Druckvermerk: Christian Heinrich Kleukens Druck der Ernst Ludwig Presse zu Darmstadt, September 1923. Schnitt der Initialen durch Oskar Becker. Zweihundertfünfzig numerierte Abzüge, davon 50 auf van Gelder Bütten. Nr. ...

[Die Exemplare für die Soncino-Gesellschaft haben einen Zusatz zum Druckvermerk:] Fünfzig Exemplare wurden als Sonderpublikation für die Mitglieder der Soncino-Gesellschaft der Freunde des jüdischen Buches hergestellt. Horodisch 16, Heider 16

18

Der Prophet Jona. Darmstadt: Kleukens, 1924. 13 Seiten.

Druckvermerk: Zweiter in der Judith-Type hergestellter Druck der Ernst Ludwig Presse. Übertragung von Martin Luther. Holzschnitte von Antes. Erschienen im Kleukens Verlag zu Darmstadt, Dezember 1924, in einer einmaligen Auflage von zweihundertfünfzig Abzügen. Nr. ...

Von diesem Werk wurden einhundert Exemplare und zwar die Nummern 41 bis 90 und 191 bis 240 als erste Sonderpublikation der Soncino-Gesellschaft der Freunde der jüdischen Buches e.V. Berlin ausgegeben. Horodisch nicht verzeichnet, Heider nicht verzeichnet

19

Die Pessach-Haggadah des Gershom Kohen Prag 5287/1527. Faksimile und Beiheft. Berlin 1926. Faksimile: 38 Blätter, Beiheft: 15 Seiten.

Druckvermerk im Beiheft: Zweite Sonderpublikation der Soncino-Gesellschaft der Freunde des jüdischen Buches e.V., Berlin. Der Faksimile-Druck der von Gerschom Kohen zu Prag 5287 (1527) gedruckten Haggadah wurde als 1. Band der im Verlage Josef Altmann, Berlin, erscheinenden Monumenta Hebraica et Judaica, fontes historiae literarum typographiae hebraicarum von Benzion Katz und Heinrich Loewe herausgegeben. Für die Mitglieder der Soncino-Gesellschaft wurden neben der allgemeinen Ausgabe 300 Exemplare auf van Geldern-Holland-Bütten gedruckt. 5686 / 1926. Horodisch 17, Heider 17 
20

Denkmal der Freundschaft. Stammbuchblätter und Widmungen von Moses Mendelssohn. Ausgewählt und herausgegeben von Fritz Bamberger. Berlin 1929. 37 Blätter.

Druckvermerk: Dritte Sonderpublikation der Soncino-Gesellschaft der Freunde des jüdischen Buches e.V., Berlin. Gesetzt aus der Unger-Fraktur und in 350 Exemplaren hergestellt durch Aldus Druck Berlin. Im Mendelssohn-Jahr 1929. Horodisch 18, Heider 18

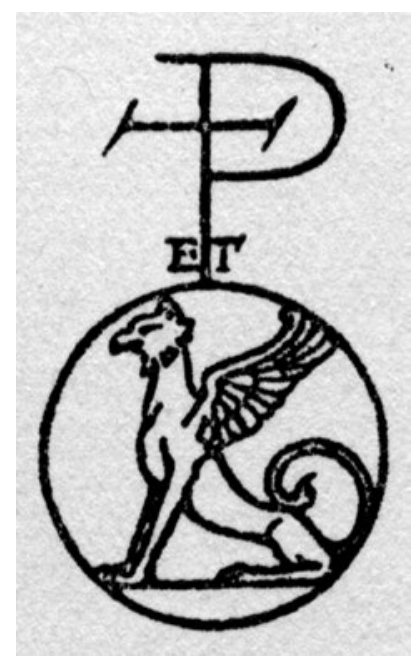

Abb. 41: Druckerzeichen Poeschel \& Trepte.

\section{Zeitschriften}

\section{1}

Soncino-Blätter. Beiträge zur Kunde des jüdischen Buches. Herausgegeben von der Soncino-Gesellschaft der Freunde des jüdischen Buches e.V. Redaktion: Herrmann Meyer. 1925-1935 [1937] 4 Bände. Horodisch 19 (Band I-III), Horodisch $19 a$ (Band IV), Heider 19 (Band I-IV)

I. Band / Berlin 1925-1926. 266 Seiten in 2 Bänden erschienen. [Band I/1:] 1. Jahrgang / Heft 1-2 / Oktober 1925. 151 Seiten.

Druckvermerk: Otto von Holten, Berlin. 
[Band I/2:] I. Band / Berlin 1925-1926. 152-266 Seiten.

Druckvermerk: Im Auftrag der Soncino-Gesellschaft der Freunde des jüdischen Buches e. V., Berlin wurde dieses zweite Doppelheft, das den ersten Band der Soncino-Blätter abschließt, im Jahre 1926 bei Poeschel \& Trepte in Leipzig aus der Fleischmann-Antiqua gesetzt und in 800 Exemplaren gedruckt.

II. Band / Berlin 1927. 194 Seiten.

Druckvermerk: Gedruckt bei Poeschel \& Trepte in Leipzig.

III. Band / Berlin 1929-1930. 79/182 Seiten in zwei Bänden erschienen. [Band III/1:] Band 3, Juli 1929, 1. Heft. 79 Seiten.

Druckvermerk: Aldus Druck Berlin.

[Band III/2:] Band 3, Juli 1930, 2.-4. Heft.

Festschrift für Heinrich Brody. Herausgegeben von Ismar Elbogen, Aron Freimann, Hermann Pick, David Simonsen. Berlin 1930. VIII, 182 Seiten.

Druckvermerk: Erscheint als Sonderheft der Soncino-Blätter. Beiträge zur Kunde des jüdischen Buches. Herausgegeben von der Soncino-Gesellschaft der Freunde des jüdischen Buches e.V. unter der Redaktion von Herrmann Meyer. Aldus Druck Berlin.

[Band IV] Festschrift für Aron Freimann zum 60. Geburtstage. Herausgegeben von Alexander Marx und Herrmann Meyer. Dargebracht von der Soncino-Gesellschaft der Freunde des jüdischen Buches. Berlin 1935 [1937]. 175 Seiten. Druckvermerk: Aldus Druck Berlin.

22

Soncino-Nachrichten. Beilage zu den Soncino-Blättern, Band I, Berlin 1925-1926. Berlin 1927. 20 Seiten.

[Enthält das] Mitgliederverzeichnis nach dem Stand vom 1. Februar 1927.

Druckvermerk: Gedruckt bei Poeschel \& Trepte in Leipzig. Horodisch nicht verzeichnet, Heider 20 
Mitteilungen der Soncino-Gesellschaft. Verantwortl.: Dr. A. Horodisch in Berlin. Druck: Aldus Druck Berlin-Schöneberg. Berlin Nr. 1.1928-10.1932, Neue Folge Heft 1.1932-Heft 2.1932. Horodisch 20, Heider 21

Nr. 1 / März 1928. 8 Seiten.

Nr. 2 / April 1928. 8 Seiten.

Nr. 3 / Juli 1928. 40 Seiten.

Nr. 4 / Februar 1929. 16 Seiten.

Nr. 5 / August 1930. 32 Seiten.

Nr. 6 / September 1930. 32 Seiten.

Nr. 7-10 / März 1931. 120 Seiten.

Neue Folge / Heft 1 / Mai 1932. Seite 1-32.

Neue Folge / Heft 2 / Oktober 1932. Seite 33-64.

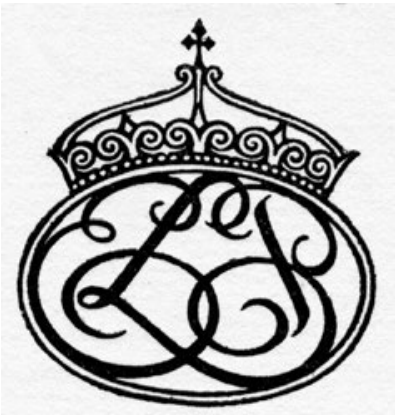

Abb. 42: Druckerzeichen Ernst Ludwig Presse.

\section{Gelegenheitsdrucke}

24

Holzschnitte aus dem Zemach Zadik, einem in Venedig im Jahre 1600 erschienenen hebräischen Moralbüchlein. Berlin 1924. 2 Blätter mit 39 Holzschnitten. [Der Druckvermerk ist der Titel]

Druckvermerk: Den Teilnehmern an der am 31. August 1924 in Berlin tagenden Sitzung des Zentralkomitees der Z.V.F.D. überreicht die Soncino-Gesellschaft der Freunde des jüdischen Buches diesen Vorabdruck der für die erste Gesellschaftsveröffentlichung bestimmten Holzschnitte aus dem Zemach Zadik, einem in Venedig im Jahre 1600 erschienenen Moralbüchlein. Gedruckt in 150 Exemplaren bei Otto von Holten in Berlin. Horodisch 21, Heider 22 
25

(Albert Einstein.) Gelegentliches von Albert Einstein. Zum fünfzigsten Geburtstag 14. März 1929 dargebracht von der Soncino-Gesellschaft der Freunde des jüdischen Buches zu Berlin. Berlin 1929. 34 Seiten. [Enthält ein Portrait Albert Einsteins als Frontispiz und eine Handschriftenprobe]

Druckvermerk: Auswahl des Textes und Ausstattung des Buches besorgte Abraham Horodisch im Einvernehmen mit der Familie Einstein, der für freundliche Überlassung zahlreicher unveröffentlichter Briefe Dank gebührt. Titelphotographie von Raja Kissin, Einbandvignette von Kurt Harald Isenstein. Gedruckt von Aldus Druck, Berlin, in einmaliger Auflage von 800 Exemplaren als Privatdruck für die Mitglieder der Soncino-Gesellschaft der Freunde des jüdischen Buches auf Veranlassung einiger Freunde der Gesellschaft. Erschienen im März 1929 in Berlin. Horodisch 22, Heider 23

26

Soncino-Gesellschaft. Hebräisch von Marcus Behmer. [Berlin 1929]. 2 Doppelblatt.

Druckvermerk: Gedruckt als Manuskript in 25 Exemplaren. Weitergabe verboten. Horodisch 28, Heider 24

27

[Bereshit] Probe der hebräischen Bibel. Schrift und Initialen von Marcus Behmer. Druck von E.W. Tieffenbach auf der Handpresse der Officina Serpentis. SoncinoGesellschaft der Freunde des jüdischen Buches. e.V. Berlin 1929. 4 Blätter. Horodisch 29, Heider 25

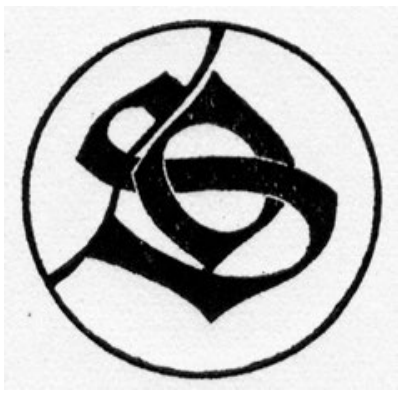

Abb. 43: Druckerzeichen Officina Serpentis / Tieffenbach. 


\section{Werbe- und ausgewählte Vereinsdrucksachen}

\section{8}

Soncino-Gesellschaft der Freunde des jüdischen Buches e.V. Berlin. Berlin 1924. 17 Blätter.

[Erstes Werbeheft der Gesellschaft:] Ehren-Ausschuss / Zur Begründung der Soncino-Gesellschaft / [Zwei Vorabdrucke:] Aus dem Roman „Zurückgekehrt“ von Samuel Lewin. Die Soncino-Gesellschaft bringt den bisher unveröffentlichten jüdischen Roman in deutscher Übersetzung als ihre zweite Publikation / Aus dem Aufsatz „Das Schreiben der Sefer Thora“ von Prof. Dr. Ludwig Blau, Budapest. Der vollständige Aufsatz erscheint im ersten Heft der Soncino-Blätter, das Ende des Jahres 1924 zur Ausfertigung kommt / Auszug aus den Satzungen der Soncino-Gesellschaft. Interessenten erhalten Abdruck der vollständigen Satzung auf Verlangen von der Soncino-Gesellschaft / Vorstand. / Lose Beilage: Aufnahmeantrag]

Druckvermerk: Dieses Werbeheft wurde im Herbst 1924 von Poeschel \& Trepte in Leipzig gedruckt. Die Klischees der beigegebenen Druckerzeichen wurden von der graphischen Kunstanstalt Albert Frisch in Berlin angefertigt. Das auf dem Titelblatt befindliche Signet der Gesellschaft ist in Anlehnung an das Druckerzeichen des Gerson Soncino für die Soncino-Gesellschaft geschaffen. Horodisch 23, Heider 27

\section{9}

Soncino-Gesellschaft der Freunde des jüdischen Buches e.V. Geschäftsstelle: Berlin C 2, Kaiser-Wilhelm-Strasse 12. Postscheckkonto: Direktor M. Simon (Schatzmeister der Soncino-Gesellschaft) Berlin Nr. 117546. Signet. Berlin 1924. 29 Seiten.

Druckvermerk: Das erste Mitgliederverzeichnis der Soncino-Gesellschaft wurde für die bis zum 15. Oktober 1924 beigetretenen Mitglieder in einer Auflage von 300 Exemplaren hergestellt. Den Druck besorgte Otto von Holten, Berlin. Horodisch 24, Heider 28

\section{0}

Soncino-Blätter. Beiträge zur Kunde des jüdischen Buches. Herausgegeben von der Soncino-Gesellschaft der Freunde des jüd. Buches. Schriftleitung: Prof. A. Freimann und Arnold Zweig. Redaktion. Berlin C 2, Kaiser-Wilhelm-Straße 12. Vertrieb: Otto von Holten, Berlin C 19, Neue Grünstrasse 13. [1924] 1 Blatt. [Maschinenschriftlicher Brief mit dem zitierten Briefkopf und der Signatur von Arnold Zweig. Datiert 24. Oktober 1924. Einladungsbrief zur Mitarbeit an den Son- 
cino-Blättern. Serienbrief ohne Adressaten (Sehr geehrter Herr!)]. Horodisch nicht verzeichnet, Heider 29

31

Soncino-Gesellschaft der Freunde des jüdischen Buches e.V. Geschäftsstelle: Berlin C 2, Kaiser-Wilhelm-Strasse 12. Postscheckkonto: Direktor M. Simon (Schatzmeister der Soncino-Gesellschaft) Berlin Nr. 117546. Berlin [1924]. 1 Doppelblatt.

[Maschinenschriftlicher Brief mit dem zitierten Briefkopf, unterzeichnet und mit Zionsgruß versehen von Herrmann Meyer und Siegfried Wolff. Werbebrief vor allem an Mitglieder zionistischer Organisationen. Angehängt die gedruckte Wiedergabe des Textes „Die Soncino-Gesellschaft“ von Arnold Zweig aus der „Jüdischen Rundschau“ 51 vom 27. Juni 1924 und eine Namensliste der Mitglieder des Vorstandes und des Ehrenausschusses der Gesellschaft] Horodisch nicht verzeichnet, Heider 31

32

Soncino-Gesellschaft der Freunde des jüdischen Buches e.V. (...). „An die Mitglieder der Soncino-Gesellschaft“. Berlin 1925. 1 Doppelblatt.

[Rundbrief an die Mitglieder vom 22. März 1925. Enthält kurze Informationen unter folgenden Überschriften: Publikationen, Zeitschriften, Sonderpublikationen, Einbände, Schriftwechsel, Sprechzeit, Mitgliederzahl, Jahresversammlung. Als 1. Sonderpublikation wird hier nicht „Das Buch Judith“ (s.o. Nr. 16), sondern „Das Buch Jona“ aus der Kleukens-Presse angekündigt]. Horodisch nicht verzeichnet, Heider 32

33

Soncino-Blätter. Beiträge zur Kunde des jüdischen Buches. Herausgegeben von der Soncino-Gesellschaft der Freunde des jüdischen Buches. 1. Jahrgang Berlin 1925. Berlin 1925. 2 Blätter.

[Ankündigung des ersten Jahrgangs]

Druckvermerk: Otto von Holten, Berlin C. Horodisch nicht verzeichnet, Heider 30

34

Soncino-Gesellschaft der Freunde des jüdischen Buches. Bericht über die Tätigkeit im ersten Gesellschaftsjahr. Berlin 1925. 55 Seiten.

Druckvermerk: Der erste Tätigkeitsbericht der Soncino-Gesellschaft wurde im Sommer 1925 (5685) bei Marx \& Co., Berlin, in 800 Exemplaren gedruckt. Horodisch 25, Heider 33 
35

Soncino-Gesellschaft der Freunde des jüdischen Buches e.V. Berlin 1925. 11 Blätter. [Aufnahmeformular als Beilage]

Druckvermerk: Das zweite Werbeheft der Soncino-Gesellschaft der Freunde des jüdischen Buches e.V. zu Berlin wurde im Herbst des Jahres 1925 in der von Rudolf Koch gezeichneten Antiqua bei Otto von Holten in Berlin gedruckt und zu Rosch Haschana 5686 (19. September 1925) fertiggestellt. Das auf dem Umschlagtitel abgedruckte Signet der Gesellschaft wurde in enger Anlehnung an das Druckerzeichen des Gerson Soncino für die Soncino-Gesellschaft geschaffen. Horodisch 26, Heider 34

36

Soncino-Gesellschaft. Mitgliedskarte für das Jahr 1926. [Ohne Ort] 1926. 1 Blatt. [Der Avers mittig mit dem Turmsignet, flankiert von Namen der Gesellschaft, links in deutscher, rechts in hebräischer Version, oben das Wort „Mitgliedskarte“, unten „für das Jahr 1926“. Verso die Anschrift der Gesellschaft und Raum für den Namen des Mitgliedes. Schwarzer Druck auf weißem Grund. Zeichnung von Josef Budko] Horodisch nicht verzeichnet, Heider 26

37

Postkarte an die Soncino-Gesellschaft e. V. Berlin C 2, Kaiser-Wilhelm-Str. 12. [Ohne Ort] 1927. Horodisch nicht verzeichnet, Heider nicht verzeichnet

[Bestellvordruck für Publikationen]

38

Soncino-Gesellschaft der Freunde des jüdischen Buches e.V. Berlin. 1 Doppelblatt. [ohne Ort] [ohne Jahr] Horodisch nicht verzeichnet, Heider nicht verzeichnet [Vordruck für Beitrittserklärung mit Auszug aus den Satzungen]

\section{9}

Der Vorstand der Soncino-Gesellschaft der Freunde des jüdischen Buches e.V. gibt sich die Ehre (...). Einladung zum Mitgliederabend am 5. September 1929. [Ohne Ort] 1929. 1 Doppelkarte.

[Der Abend war dem Andenken Moses Mendelssohns zum 200. Geburtstag gewidmet. Front mit dem Schattenriß Mendelssohns, innen der Text der Einladung in Rot und Schwarz sowie das Programm des Abends und die Rednerfolge (Fritz Bamberger, Karl Schwarz, Martha Goldberg, Willi Dorn, Herrmann Meyer). Datiert Berlin, den 30. August 1929]

Druckvermerk: Aldus Druck, Berlin. Horodisch nicht verzeichnet, Heider 35 
40

Soncino-Gesellschaft der Freunde des jüdischen Buches e.V. Einladung zur Tagung. Frankfurt am Main, 24. bis 26. Mai 1931. Berlin 1931. 4 Blätter.

Druckvermerk: Aldus Druck Berlin. Horodisch nicht verzeichnet, Heider 36

41

Herrn ... in Anerkennung für seine erfolgreiche Mitarbeit bei der Vorbereitung und Durchführung der Tagung unserer Gesellschaft in Frankfurt am Main den XXIV.-XXVI. Mai MCMXXXI. Berlin, den XXVIII. Mai MCMXXXI. Der Vorstand der Soncino-Gesellschaft der Freunde des jüdischen Buches. [Ohne Ort] 1931. 4 Blätter.

[Mit dieser Auszeichnung wurden Edwin Baer, Heinrich Eisemann, Richard Ettinghausen. Aron Freimann, Felix Kaufmann, Max Levy, Ludwig Riess, Siegfried Rosenberg bedacht] Horodisch nicht verzeichnet, Heider 37

42

Soncino-Gesellschaft der Freunde des jüdischen Buches e.V. [Ohne Ort] 1931. 2 Blätter plus 1 Anmeldepostkarte. [Enthält eine Liste aller Veröffentlichung bis zu diesem Zeitpunkt]

Druckvermerk: „Anbei überreichen wir unseren Mitgliedern mit den besten Wünschen zum Neuen Jahr vier Veröffentlichungen, die den Teilnehmern an der Jahresversammlung 1931 gewidmet waren und von denen auf unsere Veranlassung zweite Auflagen an die Gesamtheit unserer Mitglieder hergestellt werden konnten. ...“. Horodisch nicht verzeichnet, Heider nicht verzeichnet

43

Soncino-Gesellschaft der Freunde des jüdischen Buches e.V. Berlin C 2, KaiserWilhelm-Str. 12, Telephon: D 1 Norden (4447). Berlin 1931. 2 Blätter.

[Schreiben an die Mitglieder, Bitte um Überweisung des Jahresbeitrages] „Wie Ihnen bekannt ist, hat unsere Gesellschaft durch die Vorbereitung des Monumentaldruckes der hebräischen Bibel gegenwärtig besonders große Verpflichtungen....". Horodisch nicht verzeichnet, Heider nicht verzeichnet

44

Soncino-Gesellschaft der Freunde des jüdischen Buches e.V. Berlin / im Februar 1932. Aldus Druck Berlin. 4 Blätter.

„Unseren Mitgliedern soll im Jahre 1932 ein besonders reichhaltiges und wertvolles Publikationsprogramm geboten werden...“. [Liste der Veröffentlichungen] Horodisch nicht verzeichnet, Heider nicht verzeichnet 
45

Soncino-Gesellschaft der Freunde des jüdischen Buches e.V. Berlin im Februar 1932. 4 Blätter. [Mitgliederrundschreiben mit der Ankündigung der geplanten Publikationen, einem Aufruf zur Mitgliederwerbung, einer Liste noch vorhandener Publikationen und Kontaktdaten. Die Bauchbinde ist mit der Aufforderung zur Zahlung des fälligen Jahresbeitrages versehen]

Druckvermerk: Druck von Aldus Druck Berlin. Horodisch nicht verzeichnet, Heider 38

46

Soncino-Gesellschaft der Freunde des jüdischen Buches e.V. Berlin C 2. Berlin [1934]. 8 Blätter plus ein am Rand perforiertes Aufnahmeformular. [Drittes und letztes Werbeheft der Soncino-Gesellschaft]

Druckvermerk: Gesetzt aus Didot-Antiqua und -Kursiv und gedruckt bei Aldus Druck Berlin. Horodisch 27, Heider 39

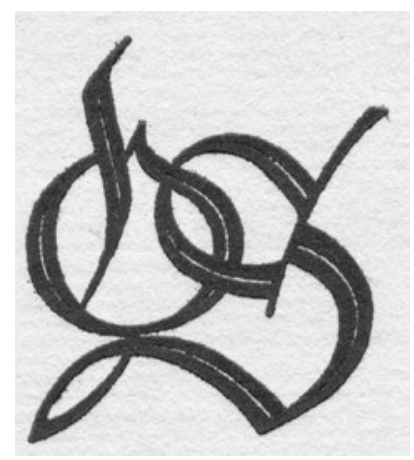

Abb. 44: Druckerzeichen Officina Serpentis / Tieffenbach.

\section{Spendendrucke}

47

Hermann Struck. Beim Studium [Berlin 1924]. 2 Blätter. [Doppelblatt mit einer Lithographie von Struck]

Druckvermerk: Den Teilnehmern an der konstituierenden Sitzung der ,SoncinoGesellschaft‘ Berlin 15. Mai 1924 / 11. Ijar 5684 gewidmet von L.L. hergestellt in 50 Exemplaren. Dieses ist Exemplar No. ... Horodisch nicht verzeichnet, Heider 40 
48

Machsor [Auszug]. Berlin 1924. 3 Blätter.

Druckvermerk: Wiedergabe aus der Editio Princeps des Machsors, gedruckt von den Mitgliedern der Familie Soncino zu Soncino und Casalmaggiore in den Jahren 1486-87. In fünfzig Abzügen für die Teilnehmer an der Gründungs-Versammlung der Soncino-Gesellschaft hergestellt. Berlin, den 11. Ijar 5684/15. Mai 1924. [Gespendet von Moses Marx und Abraham Horodisch]. Horodisch 30, Heider 41

49

S. J. Agnon. Ma'ase Rabbi Gadiel ha-Tinoq (Geschichte Rabbi Gadiels des Däumlings). Berlin [5]686 (1925). 8 Blätter. [Hebräisch]

Druckvermerk [Hebräisch, deutsche Übersetzung nach Horodisch]: „Die Geschichte vom Rabbi Gadiel dem Däumling“ erschien zum ersten Mal in Bd. 4 von „Miqlat“, New York, Elul [5]680 [=1920]. Die vorliegende Ausgabe ist in 500 Exemplaren gedruckt, als Gabe für die Mitglieder der Soncino-Gesellschaft anlässlich ihrer ersten Jahresversammlung am 1. Sivan [5]652 [=24. Mai 1925] von der Druckerei Marx \& Co., Berlin. Horodisch 31, Heider 42

50

[Klappkarte Vorderseite] Soncino-Gesellschaft der Freunde des juedischen Buches e.V., 24. Mai 1925, [Berlin 1925], 1 Klappkarte, 16 x 12,5 cm, ebenfalls als zwei Einzelkarten.

[Klappkarte rechte Innenseite] Festessen aus Anlass der ersten Jahresversammlung, Speisenfolge: Bouillon mit Mark, Rheinlachs mit Remoulade, Pökelzunge und Rinderbrust garniert, Wiener Apfelstrudel.

[Klappkarte Rückseite] Druckvermerk: Das Diner (sic!) ist von dem unter Aufsicht der Gemeinde Adass Jisroel stehenden Restaurant „Vienna“ Kantstr. 133 geliefert. Den Teilnehmern am Festmahl widmet diese von Josef Budko gezeichnete Karte Felix Struck. [Druck:] Marx \& Co., Berlin. Horodisch nicht verzeichnet, Heider 43

51

Willy Auerbach. [Gelegenheitsgedicht] (Szefiroh-Melodie). Berlin 1925. 1 Blatt. [10-strophiges Geburtstagsgedicht auf die Mitglieder des Vorstandes der SoncinoGesellschaft]

Druckvermerk: Zur ersten Geburtstagsfeier der Soncino-Gesellschaft am 1. Siwan 5685 (24. Mai 1925) widmet diesen bei Otto v. Holten, Berlin, in 150 Exemplaren 
hergestellten Gelegenheitsdruck den Teilnehmern am Festmahl Willy Auerbach. Horodisch 31a, Heider 44

\section{2}

[Biblia Hebraica] Schemot K‘. Exod. XX. Berlin 1925. 2 Blätter.

Druckvermerk: Societati honestissimae Soncino ad diem primi conventus solemniter celebrandum. Berolini. D. prim. mens. siv. VMDCLXXXV ab creatione mundi. Dedicaverunt Typographaeum Drugulini. Riccardus Handl. Impressum Typis Druglini Lipsia CIC C CIC XXV. Horodisch 32, Heider 45

\section{3}

Ludwig Börne. Juden in der freien Stadt Frankfurt. Berlin 1925. 4 Blätter.

Druckvermerk: Den Teilnehmern an der am 24sten Mai 1925 zu Berlin tagenden ersten Jahresversammlung der Soncino-Gesellschaft der Freunde des jüdischen Buches e.V. widmen den Abdruck des hundertunddritten Absatzes aus Börnes Fragmenten und Aphorismen Siegfried Wolff und Hermann Meyer. Gedruckt in 150 Exemplaren bei Marx \& Co., Berlin. Horodisch 33, Heider 46

[Exemplar 151 ist ein Druckerscherz für Herrmann Meyer:] Das hundertundeinundfünfzigste Exemplar wurde als einziges auf echt Kaiserlich von Zanders-Japan für Hermann MZ Meyer gedruckt und ihm beim Festessen anlässlich der Pökelzunge überreicht. Horodisch nicht verzeichnet, Heider $46 a$

\section{4}

Jeschuben Elieser ben Sirah. Sefer Hakma, 42, 15-25. Leipzig 1925. 4 Blätter. [Aus dem Buch:] Das Buch Sirach, von dem ich hier Kapitel 42, Vers 15-25, den Psalm über die Herrlichkeit Gottes und seiner Werke, vorlege, gehört zu den sogenannten Weisheitsbüchern. ...Leipzig, 24. Mai 1925 J. Rodenberg]

Druckvermerk: Dieses Büchlein wurde der Soncino-Gesellschaft in Berlin anlässlich der Jahresversammlung 1925 von der Schriftgiesserei H. Berthold gewidmet. Es wurde aus Frank-Rühl-Hebräisch und Antiqua Venetia gesetzt. Der Druck erfolgte in 200 numerierten Exemplaren bei Poeschel \& Trepte in Leipzig. Das Schriftchen ist die Vorwidmung zu einer größeren Abhandlung über Hebräische Typen und Schriftarten des verstorbenen Leipziger Oberkantors Rafael Frank, die die Schriftgiesserei H. Berthold den Mitgliedern der Soncino-Gesellschaft im Herbst dieses Jahres darzubieten gedenkt. Horodisch 34, Heider 47 
55

Sefer hokmat Yeshu. Die Pessach Haggadah des Gerschom Kohen gedruckt zu Prag 5287 / 1527. Drei Tafeln aus dem Neudruck hergestellt in 150 Exemplaren. Berlin 1925. 3 Blätter.

Druckvermerk: Den Teilnehmern der 1. Jahresversammlung der Soncino-Gesellschaft, Berlin, den 24. Mai 1925 gespendet von Josef Altmann, Berlin. Horodisch 35, Heider 48

56

Volks-Scene in dem Drama Sir Thomas More. Shakespeare zugeschrieben. T.J. Cobden-Sanderson dem hervorragenden Drucker des deutschen Faust in Memoriam. Darmstadt: Ernst Ludwig Presse 1925. 19 Seiten.

Druckvermerk: Druck der Ernst-Ludwig Presse zu Darmstadt. Der Soncino-Gesellschaft in Berlin anlässlich ihrer Jahresversammlung am 24. Mai 1925 überreicht durch Christian Heinrich Kleukens den Drucker. Fünfundsiebzig Exemplare wurden hergestellt. Horodisch 38 [eigentlich 36], Heider 49

57

Von Gotts gnaden Johans Friderich / Hertzog zu Sachssen / Des heiligen Römischen Reichs Ertzmarschall und Churfürst / Landgraff in Düringen / Marggraff zu Meissen / und Burggraff zu Magdeburg. [Ohne Ort] 1925. 1 Blatt.

Druckvermerk: Das Gesamtarchiv der Deutschen Juden, Berlin, spendet zur ersten Jahresversammlung der Soncino-Gesellschaft am 24. Mai 1925 den Faksimiledruck eines Kursächsischen Erlasses vom 6. Mai 1543, der auf Luthers judengegnerisches Auftreten Bezug nimmt. Zusendung erfolgt an die Teilnehmer direkt. Horodisch 37, Heider 50

58

Wir Bürgermeister und Rath der Stadt Leipzig hiermit urkunden ... [Verordnung vom 7. März 1687 über den Handel der Juden auf der Leipziger Messe] Reproduktion. [Ohne Ort] 1925. 1 Blatt.

Druckvermerk: Der Soncino-Gesellschaft widmet aus Anlaß ihrer ersten Jahresversammlung am 24. Mai 1925 Herr Salli Kirschstein die Reproduktion einer Verordnung vom 7. März 1687 über den Handel der Juden auf der Leipziger Messe. Die Spende wird den Teilnehmern am Festessen direkt übersandt werden. Horodisch 38, Heider 51 
59

Menu zum Festessen aus Anlass der zweiten Jahresversammlung der SoncinoGesellschaft der Freunde des jüdischen Buches e.V. Berlin den 5. XII. 1926. Berlin 1926. Dreifachkarte.

[Speisefolge:] Legierte Suppe - Karpfen - Gefüllte Kalbsbrust mit Gemüsen Apfelstrudel

Druckvermerk: Das Klischee nach Zeichnung von Franziska Baruch, den Satz und Druck in 250 Exemplaren besorgte die Firma Richard Labisch \& Co. G.M.B.H., Berlin 0 27, Schicklerstrasse 5.

Den Teilnehmern überreicht von Richard Labisch, Max Lefson, Walter Metzenberg. Horodisch nicht verzeichnet, Heider 52

60

[Moritz Simon] Abramson. [Ohne Ort] 1926.

Druckvermerk: Dieser Druck wurde in dreihundert Exemplaren bei Albert Frisch, Berlin hergestellt und den Freunden und Mitgliedern der Soncino-Gesellschaft aus Anlaß der zweiten Jahresversammlung am 5. Dezember 1926 überreicht von Moritz Simon. Horodisch nicht verzeichnet, Heider 53

61

I(smar) Elbogen. Moritz Steinschneider der Vater der hebräischen Bibliographie. Von Prof. Dr. I. Elbogen, Berlin. Leipzig 1926. 5 Blätter.

Druckvermerk: Dieser Vorabdruck aus dem im Erscheinen begriffenen Heft drei bis vier der Soncino-Blätter wurde in 300 numerierten Exemplaren bei Poeschel \& Trepte in Leipzig gedruckt und den Mitgliedern und Gästen der Soncino-Gesellschaft am 5. XII. 26 überreicht. Nummer ... Horodisch 39, Heider 54

\section{2}

[Joseph ben Josua Ha-Kohen] Die Verschwörung des Fiesko zu Genua. Übersetzt von Joseph Zedner, Nachwort von Herrmann Meyer. (Berlin) 1926. 8 Blätter.

Druckvermerk: Rudolf Koch, den Fünfzigjährigen zu ehren, wurde diese Erzählung in der Maximilian-Gotisch auf der Handpresse der Officina Serpentis gedruckt und den Mitgliedern der Soncino-Gesellschaft der Freunde des jüdischen Buches am 5. Dezember 1926 überreicht von Gotthard Laske und Gustav Wisbrun.

Erweiterter Druckvermerk nur in den bezeichneten Exemplaren: 20.11.1926. Zwölf Exemplare wurden auf japanischem Büttenpapier abgezogen. Horodisch 40 (ohne Hinweis auf die 12 Exemplare auf Büttenpapier), Heider 55 
63

Conrad Photorin (d.i. Georg Christoph Lichtenberg). Timorus, das ist, Vertheidigung zweyer Israeliten, die durch die Kräftigkeit der Lavaterischen Beweisgründe und der Göttingischen Mettwürste bewogen den wahren Glauben angenommen haben. Von Conrad Photorin, der Theologie und Belles Lettres Candidaten. Herausgegeben von Herrmann Meyer. Mit einem Nachwort von Martin Domke. [Nachdruck der Ausgabe Berlin 1773] Berlin, 5. Dezember 1926. 78, XVI Seiten.

Druckvermerk: Den Teilnehmern am Festessen anläßlich der Jahresversammlung der Soncino-Gesellschaft der Freunde des jüdischen Buches am 5. Dezember 1926 zu Berlin gewidmet von Reinhold und Erich Scholem. Gesamtherstellung Arthur Scholem, Berlin. Gesetzt aus der Goethe-Fraktur und Winchester Old Style auf einer Typograph-Setzmaschine. Das Titelblatt wurde faksimiliert. Das Büttenpapier stiftete die Papiergroßhandlung S.L. Cahen, Berlin. Gedruckt in 222 Exemplaren, welche mit der Hand numeriert sind. Horodisch 41, Heider 56

64

(Heinrich Loewe.) In Memoriam Aaron Ember. (Berlin 1926). 4 Blätter.

Druckvermerk: Dem Andenken des Mitgliedes der Soncino-Gesellschaft Aaron Ember und seiner Frau Regina, geb. Mandelstamm zur zweiten Jahresversammlung der Gesellschaft gewidmet von Frieda und Hermann Stahl. 29. Kislew 5687. Copyright by Prof. Dr. Heinrich Loewe, Berlin 1926. Horodisch 42, Heider 57

65

(Heinrich Loewe.) Proselyten. Ein Beitrag zur Geschichte der jüdischen Rasse von Heinrich Loewe. Berlin 5687 (1926). 31 Seiten.

Druckvermerk: Im Auftrage von Simon Braun wurde dieses Buch bei Arthur Scholem, Berlin in dreihundert Exemplaren gedruckt und den zur Jahresversammlung der Soncino-Gesellschaft vereinigten Freunden des jüdischen Buches überreicht. 29. Kislew 5687 - 5. Dezember 1926. Copyright by Soncino-Gesellschaft e.V. Berlin 1926. Horodisch 43, Heider 58

66

Masa išamasa tob. Leipzig 1926. 5 Blätter. [Text gegenläufig in Hebräisch und Deutsch. Die Übersetzung monogrammiert H.L. = Heinrich Loewe]

Druckvermerk: Den Freundinnen des jüdischen Buches zur II. Jahresversammlung der Soncino-Gesellschaft die Offizin Richard Hadl in Leipzig MCMXXVI. Horodisch 44, Heider 59 
67

Moses Mendelssohn. Eine Stammbuch-Eintragung Moses Mendelssohns. [Ohne Ort] 1926. [Faksimile des Stammbuch-Eintrags]

Druckvermerk: Den Freunden und Mitgliedern der Soncino-Gesellschaft zum 5. Dezember 1926 gewidmet von Herrmann Meyer, 1926. Horodisch 45, Heider 60

68

Jacob Plessner. [Der Bücherfreund] Lithographie. [Ohne Ort] (1926).

Druckvermerk: Der Soncino-Gesellschaft zum 5. Dez. 1926“. [Von Jacob Plessner signiert] Horodisch 46, Heider 61

69

Porträt des hebräischen Dichters Chajim Nachman Bialik nebst seiner Biographie aus der Jüdischen Enzyklopädie in vier Bänden. [Ohne Ort] 1926.

Druckvermerk: Den Teilnehmern der Jahresversammlung der Soncino-Gesellschaft der Freunde des jüdischen Buches am 4. und 5. Dezember 1926 überreicht vom Jüdischen Verlag, Berlin NW 7, Dorotheenstraße 35. Nach einem Foto von Max Treder, Charlttbg, Knesebeckstr. 76. Horodisch 47, Heider 62

70

Rafael Frank. Über hebräische Typen und Schriftarten. Mit einem Nachwort von Dr. Jacques Adler. Herausgegeben von der Schriftgiesserei H. Berthold. Berlin: Schriftgiesserei H. Berthold, 1926. 39 Seiten.

Druckvermerk: Satz und Druck von Poeschel \& Trepte in Leipzig unter Verwendung von Antiqua-Venetia und Frank-Rühl-Hebräisch der Schriftgiesserei H. Berthold AG. Hergestellt in einer beschränkten Auflage für einen Kreis von Freunden. Ferner wurden 500 gezählte Exemplare der Soncino-Gesellschaft in Berlin zur Verfügung gestellt. Dieses Exemplar trägt die Nummer ... Horodisch nicht verzeichnet, Heider 63

71

Hans Rosenkranz. Baruch Spinoza zum 21. Februar 1927. Berlin 1927. 8 Blätter.

Druckvermerk: Siegfried Alweiss druckte diesen Aufsatz für die Teilnehmer des Spinoza-Abends, den die Soncino-Gesellschaft, Berlin, anläßlich der 250. Wiederkehr von Spinoza's Todestag veranstaltete. Horodisch 48, Heider 64 
72

Kurt Freyer. Spinoza. Führer der Irrenden. Gedenkschrift anlässlich der 250. Wiederkehr des Todestages Spinozas. 21. Februar 1927. Berlin: Horodisch \& Marx, 1927. 14 Blätter.

Druckvermerk: Gedruckt in 37 Exemplaren auf kaiserlich Japan und 395 Exemplaren auf van Gelder-Bütten von der Buchdruckerei Wilhelm Adam in Chemnitz für den Verlag Horodisch \& Marx Berlin. Dieses Exemplar trägt die Nummer ... Sonderdruck für Mitglieder der Soncino-Gesellschaft der Freunde des jüdischen Buches. Horodisch nicht verzeichnet, Heider 65

73

Aus der Offenbacher Haggadah. Privatdruck. Offenbach am Main 1927.

Jessen-Schrift von Rudolf Koch, Holzschnitte von Fritz Kredel, Typographie von Max Dorn, sämtlich in Offenbach am Main. 6 Blätter. Seiten 1-8.

Druckvermerk: Zur 6. Jahresversammlung der Soncino-Gesellschaft gewidmet von Dr. Guggenheim in Offenbach am Main. Horodisch nicht verzeichnet, Heider 66

74

Aus der Offenbacher Haggadah. Privatdruck. Offenbach am Main 1927. Jessen-Schrift von Rudolf Koch, Holzschnitte von Fritz Kredel, Typographie von Max Dorn, sämtlich in Offenbach am Main. 6 Blätter. Seiten 11-24.

Druckvermerk: Zur 6. Jahresversammlung der Soncino-Gesellschaft gewidmet von Dr. Guggenheim in Offenbach am Main. Horodisch nicht verzeichnet, Heider nicht verzeichnet

75

Aus der Offenbacher Haggadah. Privatdruck. Offenbach am Main 1927. JessenSchrift von Rudolf Koch, Holzschnitte von Fritz Kredel, Typographie von Max Dorn, sämtlich in Offenbach am Main. 6 Blätter. Seiten 57-64.

Druckvermerk: Zur 6. Jahresversammlung der Soncino-Gesellschaft gewidmet von Dr. Guggenheim in Offenbach am Main. Horodisch nicht verzeichnet, Heider 67

76

Aus der Offenbacher Haggadah. Privatdruck. Offenbach am Main 1927. JessenSchrift von Rudolf Koch, Holzschnitte von Fritz Kredel, Typographie von Max Dorn, sämtlich in Offenbach am Main. 6 Blätter. Seiten 65-72. 
Druckvermerk: Zur 6. Jahresversammlung der Soncino-Gesellschaft gewidmet von Dr. Guggenheim in Offenbach am Main. Horodisch nicht verzeichnet, Heider 68

\section{7}

Aus der Offenbacher Haggadah. Privatdruck, Offenbach am Main 1927. JessenSchrift von Rudolf Koch, Holzschnitte von Fritz Kredel, Typographie von Max Dorn, sämtlich in Offenbach am Main. Offenbach am Main 1927. 6 Blätter. Seiten 81-88.

Druckvermerk: Zur 6. Jahresversammlung der Soncino-Gesellschaft gewidmet von Dr. Guggenheim in Offenbach am Main. Horodisch 49, Heider 69

78

Sophia Solomonos [Griech]. Die Weisheit Salomons. Berlin 1928. 4 Blätter.

Druckvermerk: Griechischer Text der Cambridge Ausgabe, deutsche Übersetzung von K. Siegfried aus der Bibelübersetzung von Kautzsch. Dritte Jahresversammlung der Soncino-Gesellschaft 20.5.1928.

Handpressendruck der Officina Serpentis, Steglitz, von E.W. u. E.H. Tieffenbach, in 120 Exemplaren auf chinesischem Papier. Horodisch 50, Heider 70

\section{9}

Hermann Cohen. Ausgewählte Stellen aus unveröffentlichten Briefen. Berlin 1929. 8 Blätter.

Druckvermerk: Ausgewählt und mit gütiger Erlaubnis von Frau Geheimrat Cohen herausgegeben von Dr. Bruno Strauß. Den Teilnehmern an der Jahresversammlung der Soncino-Gesellschaft der Freunde des jüdischen Buches am 17. Februar 1929 überreicht vom Akademie-Verlag. Die Auflage beträgt 150 numerierte Exemplare. Druck: Aldus Druck Berlin, Exemplar Nr. ... Horodisch 51, Heider 71

\section{0}

Vinzenz Fettmilch Todesgang. Nach einem gleichzeitigem Stich. Berlin 1929. 2 Blätter mit eingeklebter Reproduktion des Stiches.

Druckvermerk: Den Mitgliedern und Freunden der Soncino-Gesellschaft überreicht zum 17. Febr. 1929 diese Wiedergabe eines seltenen Blattes seiner Sammlung. Moritz Simon. Druck von E.W. Tieffenbach. 250 Exemplare. Horodisch 52, Heider 72 
81

(Iwan Goll.) Noemi. Berlin 1929. 5 Blätter.

Druckvermerk: $\mathrm{Zu}$ dieser Dichtung von Iwan Goll schuf Jakob Steinhardt die Holzschnitte. Aus Anlaß der Jahresversammlung der Soncino-Gesellschaft am 17. Februar 1929 gewidmet von Gotthard Laske und Abraham Horodisch. Hergestellt in einhundertfünfzig numerierten Exemplaren von Aldus Druck in Berlin. Exemplar Nr. ... Horodisch 53, Heider 73

82

(Iwan Goll.) Noemi. Berlin 1929. 5 Blätter. [Mit 3 signierten Holzschnitten von Jakob Steinhardt. Mit einer Extrasuite der Zustandsdrucke: 23 signierte und bezeichnete Holzschnitte von Steinhardt unter Passepartout]

Druckvermerk: Zu dieser Dichtung von Iwan Goll schuf Jakob Steinhardt die Holzschnitte. Gedruckt 1929 für Gotthard Laske und Abraham Horodisch. Fünfzehn numerierte Exemplare auf Japan. [Dieses Exemplar der Vorzugsausgabe mit einem gegenüber dem Spendendruck der Soncino-Gesellschaft veränderten Druckvermerk. Der Hinweis auf die Soncino-Gesellschaft fehlt] Horodisch nicht verzeichnet, Heider $73 a$

83

Jüdische Gedichte von Klopstock bis Keller. [Nachbemerkung von Abraham Horodisch] Berlin 1929. 48 Seiten.

Druckvermerk: Den am 17. Februar 1929 versammelten Mitgliedern der SoncinoGesellschaft der Freunde des jüdischen Buches überreicht von Reinhold und Erich Scholem. Gedruckt in Briefkopf-Fraktur und Didot-Antiqua bei Arthur Scholem, Berlin. 200 numerierte Exemplare. Horodisch 54, Heider 74

84

Heinrich Loewe. Geschichten von jüdischen Namen. Aus dem Volksmunde gesammelt von Heinrich Loewe. Berlin, Februar 1929. 18 Seiten.

Druckvermerk: Handpressendruck der Officina Serpentis in einer Auflage von 250 Exemplaren; den Mitgliedern und Freunden der Soncino-Gesellschaft zum Gesellschaftsabend am 17. Februar 1929 überreicht von Simon Braun. Horodisch 55a, Heider 75

85

Heinrich Loewe. Geschichten von jüdischen Namen. Aus dem Volksmunde gesammelt von Heinrich Loewe. 2. Auflage. Berlin 1929. 22 Seiten. 
Druckvermerk: Handpressendruck der Officina Serpentis. Von der ersten Auflage wurden 150 Exemplare hergestellt und den Mitgliedern und Freunden der SoncinoGesellschaft zum Gesellschaftsabend am 17. Februar 1929 von Simon Braun überreicht. Auch diese zweite Auflage, in einer Zahl von 80 Exemplaren, hat Simon Braun veranlaßt. Horodisch 55b, Heider $75 a$

\section{6}

Heinrich Loewe. Ignaz Goldziher. (Ein Wort des Gedenkens von Heinrich Loewe). Enthält einen eingeklebten Faksimile-Brief von Ignatz Goldziher. [Berlin 1929]. 6 Blätter.

Druckvermerk: Den am 7. Adar 5689 [= 17. Februar 1929] zur Jahresversammlung der Soncino-Gesellschaft vereinigten Freunden des jüdischen Buches überreicht von Josef Altmann und Buchdruckerei Max Lichtwitz. Horodisch 56, Heider 76

87

Ernst Simon. Zum Problem des jüdischen Witzes. Berlin 1929. 16 Seiten.

Druckvermerk: Handpressendruck von E.W. Tieffenbach in einer Auflage von 222 Exemplaren. Den Teilnehmern am Gesellschaftsabend der Soncino-Gesellschaft gewidmet von Gustav Wisbrun (Remscheid), Richard Goldberg (Elberfeld), Curt Munter (Berlin), Berlin den 17. Februar 1929. Horodisch 57, Heider 77

\section{8}

Gotthold Ephraim Lessing. Die Juden. Ein Lustspiel in einem Aufzuge. [Nachwort von Herrmann Meyer] Berlin 1929. 57 Seiten. [Vgl. 11 und 119]

Druckvermerk: Handpressendruck der Officina Serpentis in 200 Exemplaren. Den Mitgliedern und Freunden der Soncino-Gesellschaft zum 17. Februar 1929 gewidmet von Menko Max Hirsch. Horodisch 10a, Heider 78

\section{9}

Moses Mendelssohn in Potsdam am 30. September 1771. Eine kleine Aufhellung von Bruno Strauß. Berlin 1929. 30 Seiten.

Druckvermerk: Den Mitgliedern und Freunden der Soncino-Gesellschaft gewidmet zum 17. Februar 1929 von Herrmann Meyer. Gedruckt in 250 Exemplaren von Aldus Druck Berlin. Horodisch 58, Heider 79

90

Abraham Bedarschi. Elegie über die Konfiszierung des Talmuds in Frankreich in der ersten Hälfte des dreizehnten Jahrhunderts. Berlin 1929. 8 Blätter. 
Druckvermerk: Herausgegeben von David Rettig auf Grund der in der Bibliothek des Britischen Museums befindlichen Handschrift Add. Or. 27168 fol $64 a$ (vgl. Margoliouth, Cat. Of the Hebrew ... Ms. Part III. Nr. 930). Inhaltsangabe von Max Meyer. Privatdruck zur Festsitzung des Ehrenausschusses und Vorstandes der Soncino-Gesellschaft am 14. Mai 1929 aus Anlaß des fünfjährigen Bestehens der Gesellschaft. Gesetzt aus Didot-Antiqua und Aldus-Hebräisch und in 49 Exemplaren gedruckt durch Aldus Druck Berlin. Herrn Menko Max Hirsch dem freundlichen Gastgeber. Horodisch 59, Heider 80

91

Mendelssohn Gedenkfeier der jüdischen Gemeinde zu Berlin am 8. September 1929. Gedenkrede von Leo Baeck. Berlin 1929. 22 Seiten.

Druckvermerk auf beiliegende Karte: Vom Vorstand der Jüdischen Gemeinde zu Berlin den Teilnehmern an der Jahresversammlung 1930 der Soncino-Gesellschaft ergebenst überreicht. M. Rosenthal, Berlin SO 16. Horodisch 61, Heider 82

92

Chacham Zewi Hirsch Aschkenasi. Rechtfertigung des Rabbi David Nieto gegen den Vorwurf in seiner Predigt Spinozas Lehre zu verbreiten. [Berlin] 1930. 8 Seiten.

Druckvermerk: Dieses Responsum des Amsterdamer Rabbiners aus dem Anfange des 18. Jahrhunderts wurde als Handpressendruck der Officina Serpentis in 200 Exemplaren hergestellt und den Mitgliedern und Freunden der Soncino-Gesellschaft zum 30. März 1930 gewidmet von Curt Munter und Herrmann Meyer. Horodisch 60, Heider 81

93

Briefe berühmter Jüdinnen. Berlin 1930. 4 Brieffaksimiles in einer Mappe. [Die Mappe enthält eingelegte Blätter ohne Seitenzählung, sie besteht aus einem Faltblatt mit illustriertem Deckblatt und Nennung der Briefeschreiberinnen sowie jeweils ein Brieffaksimile von Henriette Herz, Rahel Varnhagen von Ense, Elisa Rachel, gen. Félix und Sarah Bernhardt in unterschiedlichen Formaten]

Druckvermerk: Gedruckt in 200 Exemplaren u. den Freundinnen des jüdischen Buches zur Jahresversammlung der Soncino-Gesellschaft überreicht von Gotthard Laske u. Herrm. Meyer. Berlin, den 30. März 1930. Horodisch 62, Heider 83

94

Jehuda Ha-Levi. Die schönen Versmasse. Bearbeitet von H. Brody. [Leipzig] 1930. 14 Seiten. 
Druckvermerk: Den Mitgliedern der Soncino-Gesellschaft zur Jahresversammlung 1930 gewidmet von Salmann [sic] Schocken. Gedruckt in 200 numerierten Exemplaren bei Haag-Drugulin in Leipzig. Horodisch 63, Heider 84

95

Gottlieb von Leon. Rabbinische Legenden. Berlin 1930. 45 Seiten.

Druckvermerk: Gottlieb von Leon, geboren am 17. April 1757, gestorben am 17. September 1832, war Kustos an der Wiener Hofbibliothek. Seine „Rabbinischen Legenden“ erschienen 1821 zu Wien in Carl Armbruster's Verlag. Die Erstausgabe trägt die Widmung: „Dem unvergeßlichen Andenken meiner verewigten Freunde Michael Denis, Johann Gottfried von Herder und Johann von Müller gewidmet“. Die vorliegende Neuausgabe folgt buchstabengetreu dem Erstdruck, lediglich offensichtliche Setzerfehler wurden verbessert.

Als Spende zur Jahresversammlung der Soncino-Gesellschaft der Freunde des jüdischen Buches in 800 Exemplaren gedruckt und gestiftet von M. Rosenthal, Berlin, am 30. März 1930. Gesetzt aus dem Cicerograd der Garamond-Antiqua und-Kursiv. Horodisch 64, Heider 85

96

Heinrich Loewe. Jüdischer Feuersegen. Von Heinrich Loewe. (Ein Beitrag zum jüdischen und deutschen Aberglauben). Berlin 1930. 16 Seiten.

Druckvermerk: Dieses Büchlein wurde von der Druckerei Siegfried Scholem BerlinSchöneberg, Hauptstraße 8 in dreihundert Exemplaren hergestellt und den zur Jahresversammlung der Soncino-Gesellschaft versammelten Freunden des Jüdischen Buches als Spende überreicht. 30. März 1930. Horodisch 65, Heider 86

97

Heinrich Loewe. Der Jüdische Spieler. Eine Gelegenheitsschrift von Heinrich Loewe. [Mit einer eingeklebten Abbildung eines Spielers] Berlin 1930. 12 Seiten.

Druckvermerk: Im Auftrage von Bruno Boas, Martin Brunn, Moritz Hepner und Alfred Peltesohn in der Druckerei von Siegfried Scholem, Berlin-Schöneberg in dreihundert numerierten Exemplaren hergestellt und den am 30. März 1930 zur Jahresversammlung der Soncino-Gesellschaft vereinigten Freunden des jüdischen Buches und des jüdischen Spieles als Spende überreicht. Nr. ... Horodisch 66, Heider 87 
98

Moses Maimonides. Die Dreizehn Grundlehren. 1930. 4 Blätter.

Druckvermerk: Der Soncino-Gesellschaft zum 30. III. 30. Marcus Behmer, E.W. Tieffenbach. Horodisch 67, Heider 88

99

Proben talmudischen Zivilrechtes. Vier rabbinische Responsen. [Berlin] 1930. 16 Seiten.

Druckvermerk: Der ,Soncino-Gesellschaft der Freunde des jüdischen Buches` zur Jahresversammlung 1930 als Juristengabe gewidmet von Aron Barth, M. Flörsheim, Walter Gerson, Sammy Gronemann, Leo Hirschfeld, Alfred Klee, Max Kollenscher, Eduard Leßynsky, Walter Michaelis, Alfred Platz, Max Strauß. Gesetzt und gedruckt in 200 Exemplaren von E.W. Tieffenbach auf der Handpresse der Officina Serpentis. Horodisch 68, Heider 89

100

(Gabriel Jacques von Rosenberg.) Der Hofrat erzählt jüdische Geschichten. Berlin 1930. 8 Blätter.

Druckvermerk: Dem Andenken des 1929 verstorbenen Hofrats Dr. Gabriel Jacques von Rosenberg widmen einige Freunde dieses Buch. Den Mitgliedern der SoncinoGesellschaft der Freunde des jüdischen Buches, deren Vorstand der Verstorbene lange Zeit angehörte, aus Anlaß der am 30. März 1930 zu Berlin tagenden Jahresversammlung überreicht. Gedruckt in 700 Exemplaren durch Aldus Druck Berlin. Horodisch 69, Heider 90

101

(Gabriel Jacques von Rosenberg.) Der Hofrat erzählt jüdische Geschichten. Herausgegeben von Herrmann Meyer. Berlin 1930. 16 Seiten. [Unbeschnittener Bogen] [Handschriftlicher Vermerk Herrmann Meyers:] Die Drucker zogen spaßeshalber dieses eine Expl. ab, bei dem sie, um mich zu necken, meinen Namen als Hrsg. gegen meine ausdrückliche Bitte auf das Titelblatt gesetzt hatten. Horodisch nicht verzeichnet, Heider $90 a$

102

Arnold Zweig. Die Aufrichtung der Menorah. Entwurf einer Pantomime. Nachwort von Manfred Sturmann. Versuch über Arnold Zweig. Berlin 1930. 8 Blätter. 
Druckvermerk: Gedruckt in 250 Exemplaren von Aldus Druck Berlin. Der SoncinoGesellschaft der Freunde des jüdischen Buches zur Jahresversammlung am 30. März 1930 gestiftet von Dr. Siegbert Feldberg, Stettin. Horodisch 70, Heider 91

\section{3}

Stefan Zweig. Rahel rechtet mit Gott. Legende. Mit Holzschnitten von Walter Preißer. Berlin 1930. 20 Blätter.

Druckvermerk: Gedruckt in der Breitkopf-Fraktur durch Aldus Druck, Berlin. Die Auflage beträgt 370 Exemplare. Den Mitgliedern und Freunden der Soncino-Gesellschaft zur Jahresversammlung 1930 gewidmet von Leo Heskel. Die Holzschnitte stiftete Gotthard Laske. Horodisch 71, Heider 92

104

Inhaltsverzeichnis der Festschrift Aron Freimann zum 60. Geburtstage. Dargebracht von der Soncino-Gesellschaft der Freunde des jüdischen Buches. Herausgegeben von Alexander Marx und Herrmann Meyer. Berlin 1931. 4 Blätter [inkl. Umschlag].

Druckvermerk: Diese Festschrift erscheint als Band IV der Soncino-Blätter, Beiträge zur Kunde des jüdischen Buches. Der Vorabdruck des Inhaltsverzeichnisses wurde in 20 Exemplaren auf der Handpresse der Officina Serpentis gedruckt und dem Jubilar zum 1. August überreicht. Horodisch nicht verzeichnet, Heider 93

\section{5}

(Josef Alba.) Ikkarim. [2 faksimilierte Blätter aus der Ausgabe von 1485, die Israel Natan Soncino gedruckt hatte] Frankfurt am Main 1931. 5 Blätter. [Aus dem Vorwort des hebräischen Textes von Aron Freimann: „Josef Alba's Ikkarim druckte Israel Natan Soncino und beendete den Druck am 29. Dezember 1485 in Soncino. In den Exemplaren, die die römische Zensur erreichen konnte, vernichtete sie zwei Blätter. Die Exemplare, die nach dem türkischen Reiche und dem Orient kamen, enthalten diese Blätter."]

Druckvermerk: Zur Tagung der Soncino-Gesellschaft in Frankfurt am Main am 26. Mai 1931 überreicht von Julius Werner. Von dem vorliegenden Sonderdruck wurden 200 Exemplare hergestellt, die in der Presse einzeln numeriert wurden. Dieses Exemplar trägt die Nummer ... Horodisch 72, Heider 94

\section{6}

(Julius Hülsen.) Zwei Ansichten der Frankfurter Judengasse. Mit Erläuterungen von Julius Hülsen. Als Manuskript gedruckt. Frankfurt am Main, Mai 1931. 6 Blätter, davon 2 Karten auf einem Faltblatt. 
Druckvermerk: Zur sechsten Jahresversammlung der Soncino-Gesellschaft 24. bis 26. Mai 1931 den Gästen dargebracht von Edwin Baer, Ludwig Baer, Dr. Ernst Blau, Dr. Julius Homburger, Ludwig Ries, Siegfried Rosenberg. No. ... von 100 numerierten Exemplaren. [Rückseite des Umschlags:] Druck von Kornsand \& Co., Klischees von Paul Janke, Frankfurt am Main. Horodisch 73a, Heider 95

107

(Julius Hülsen.) Zwei Ansichten der Frankfurter Judengasse. Mit Erläuterungen von Julius Hülsen. Berlin 1931. 6 Blätter, davon 2 Karten auf einem Faltblatt.

Druckvermerk: Für die Mitglieder der Soncino-Gesellschaft gedruckt in 900 Exemplaren von Kornsand \& Co., Frankfurt a.M., im Sommer 1931. Die erste Auflage dieser Schrift wurde zur 6. Jahresversammlung der Soncino-Gesellschaft 24.-26.5.1931 dargebracht von Edwin Baer, Ludwig Baer, Dr. Ernst Blau, Dr. Julius Homburger, Ludwig Ries, Siegfried Rosenberg. Horodisch 73b, Heider $95 a$

108

Bartholomaeus Kaiser. Deutsch-hebräisches Flugblatt um 1515. [Nachwort von Heinrich Eisemann] Die Unterschrift Bartholomaeus Kaisers auf dem Titelschild ist die Wiedergabe eines Teiles einer längeren eigenhändigen Eintragung des Autors in die im Text besprochene Grammatik: „Elemente Hebraicum“. Das einzige Exemplar in Halle: Bibliothek der Deutschen Morgenländischen Gesellschaft. Die Holzschnitte sind in $3 / 4$ der Originalgröße reproduziert. Die Stichätzungen lieferte die Chemiegraphische Kunstanstalt F. Guhl \& Co., Frankfurt a.M. Frankfurt am Main 1931. 7 Blätter.

Druckvermerk: Den Teilnehmern an der 6. Tagung der Soncino-Gesellschaft der Freunde des jüdischen Buches in Frankfurt am Main 24.-26. Mai 1931 gewidmet. Nach dem einzigen im „British Museum“ aufbewahrten Exemplar in zweihundertfünfzig numerierten Stücken abgezogen von denen das vorliegende die Nummer ... trägt. Druck und Ausstattung besorgte: Verlag des Israelit und Hermon G.m.b.H. Frankfurt am Main. [Horodisch nennt Eisemann als Widmenden] Horodisch 74, Heider 96

109

Heinrich Loewe. Alter jüdischer Volkshumor aus Talmud und Midrasch. Von Heinrich Loewe. (Reichenberg) 1931. 84 Seiten.

Druckvermerk: Dieses Büchlein wurde von der Druckerei Gebrüder Stiepel Ges. m.b.H. Reichenberg in Böhmen hergestellt und den Teilnehmern an der Jahresversammlung der Soncino-Gesellschaft der Freunde des jüdischen Buches von ihren Mitgliedern in der Čechoslovakischen Republik Dr. Friedrich Eckstein, Tetschen, Dr. 
Ernst Gütig, Prag, Dr. Fritz Knöpfmacher, Teplitz, Dr. Fr. Kraus, Prag, Prof. Dr. S. A. Lieben, Prag, Max Sborowitz, Prossnitz, Dr. Felix Seidemann, Teplitz, Dr. Heinz Schleim, Teplitz, Arno Stampf Teplitz, Loge Veritas Bne Brith, Saaz als Festgabe überreicht. Frankfurt am Main, 25. Mai 1931. Horodisch 75, Heider 97

110

Moses Maimonides. Des Moses Maimonides Morgengebet bevor er seine Kranken besuchte. [Berlin] 1931. 4 Blätter.

Druckvermerk: Handpressendruck der Officina Serpentis gesetzt aus der Gothik der Werkstatt; als Spende der Mediciner zur Jahresversammlung 1931 der SoncinoGesellschaft überreicht von den Doctores Medicinae Walther Bennigson, Max Eitingon, Martin Graetz, Paul Holzer, Alice Schiff, Edwin Schiller und Hermann Wollstein. Horodisch 76, Heider 98

111

Sefer k'rovot hu mahzor. [Machsor] Verlegt und gedruckt in der privilegierten oriental und occidentalischen Buchdruckerey von W. Heidenheim \& B.M. Baschwitz in Rödelheim. 1800. Frankfurt am Main 1931. 3 Blätter.

Druckvermerk: Festgabe, überreicht aus Anlaß der Tagung der Soncino-Gesellschaft in Frankfurt a.M. am 26. Mai 1931 von der Firma M. Lehrberger \& Co., Frankfurt a.M., der Nachfolgerin der von Wolf Heidenheim im Jahre 1799 in Rödelheim gegründeten Buchdruckerei und Buchhandlung. Die Festgabe stellt den Titel der im Jahre 1800 erschienenen ersten Machsorausgabe und den Cherem (Bannfluch) dar. Ein solcher Bannfluch wurde gegenüber dem ausgesprochen, der es wagen sollte, das Werk ohne Genehmigung des Verfassers nachzudrucken.

Der vorliegende Sonderdruck wurde in einer Auflage von 300 Stücken hergestellt, die in der Presse einzeln numeriert wurden. Dieses Exemplar trägt die Nummer ... Horodisch 77, Heider 99

112

Manfred Sturmann. Die Schöpfung. Berlin 1931. 8 Blätter.

Druckvermerk: Der Soncino-Gesellschaft zur Tagung in Frankfurt am Main am 25. bis 27. Mai 1931. Satz und Druck der Officina Serpentis, Berlin-Steglitz. Horodisch 78, Heider 100

113

[Leopold] Zunz. Das Buch Zunz. Künftigen ehrlichen Leuten gewidmet. Eine Probe eingeleitet und herausgegeben von Fritz Bamberger. [Berlin] 1931. 30 Seiten. 
Druckvermerk: Im Auftrag von Salman Schocken für die Soncino Gesellschaft gesetzt und gedruckt auf der Handpresse der Officina Serpentis. 26.5.1931. Horodisch 79, Heider 101

114

Zwei Flugblätter den „Klever Getstreit“ betreffend. Frankfurt am Main 1931. 4 Blätter. [Unterschiedliche Formate]

Druckvermerk: Zur Tagung der Soncino-Gesellschaft in Frankfurt am Main am 26. Mai 1931. Überreicht von Prof. Dr. Aron Freimann und Rechtsanwalt Abraham Horovitz. Horodisch 80, Heider 102

115

Zwanzig Jahre Officina Serpentis. Katalog der Ausstellung in der Staatlichen Kunstbibliothek. E.W. Tieffenbach. [Berlin] 1931. 11 Blätter.

Druckvermerk: Handpressedruck der Officina Serpentis in 125 Exemplaren für die Teilnehmer an der Besichtigung der Ausstellung durch die Mitglieder der drei Berliner bibliophilen Gesellschaften Berliner Bibliophilen Abend, Soncino-Gesellschaft und Fontane-Abend. 30. Nov.1931. Horodisch nicht verzeichnet, Heider 104

116

Jean Paul und Emanuel Osmund. Briefe über den Talmud. [Nachwort von Dr. Karl Schönberg] [Berlin] 1932. 24 Seiten.

Druckvermerk: Den zur Jahresversammlung 1932 in Berlin versammelten Mitgliedern und Freunden der Soncino-Gesellschaft widmen 150 Exemplare dieses Handpressedrucks der Officina Serpentis: Dr. Siegbert Feldberg, Dr. Moritz Garbaty, Leo Heskel, Dr. Hans Lewenz, Georg Lipschitz und Dr. Alfred Platz. Horodisch 82, Heider 103

117

Tehillim 92 [Hebräisch]. Berlin 1932. 4 Blätter.

Druckvermerk: Der zweiundneunzigste Psalm wurde den Mitgliedern der Soncino-Gesellschaft anlässlich des Festabends am 9. April 1932 in Berlin von Georg Lipschitz gewidmet. Gedruckt in 700 Exemplaren bei Aldus Druck Berlin. Schrift: Aldus-Hebräisch. Horodisch 81, Heider 105 
118

Privatdrucke von und für Gotthard Laske. [Berlin] 1932. 8 Blätter.

Druckvermerk: Handpressendruck der Officina Serpentis in 250 Exemplaren auf van Gelder- Zonen zum 50. Geburtstag von Gotthard Laske. 3. März 1932. Der Soncino-Gesellschaft zum 9. IV.1932. Horodisch 83, Heider 106

\section{9}

Gotthold Ephraim Lessing. Die Juden. Ein Lustspiel in einem Aufzuge. Berlin 1929. 2 Blätter. [Vgl. 11 und 88]

Druckvermerk: Herr Menko Max Hirsch hatte die Absicht den Teilnehmern am Gesellschafts-Abend der Soncino-Gesellschaft das Lustspiel des jungen Lessing „Die Juden“ mit einem Nachwort über die Wirkung des Werkes in seiner Zeit zu überreichen. Die zur Verfügung stehende Zeit reichte leider nicht aus einen reinen Pressendruck fertig zu stellen; wir übergeben somit zunächst nur beiliegenden Bogen als Probe. Horodisch nicht verzeichnet, Heider nicht verzeichnet 